\title{
Lifshitz scaling, microstate counting from number theory and black hole entropy
}

\author{
Dmitry Melnikov, ${ }^{a, b}$ Fábio Novaes, ${ }^{a}$ Alfredo Pérez $^{c}$ and Ricardo Troncoso ${ }^{c}$ \\ ${ }^{a}$ International Institute of Physics, Federal University of Rio Grande do Norte, \\ Av. Odilon Gomes de Lima 1722, Capim Macio, Natal-RN 59078-400, Brazil \\ ${ }^{b}$ Institute for Theoretical and Experimental Physics, \\ B. Cheremushkinskaya 25, Moscow 117218, Russia \\ ${ }^{c}$ Centro de Estudios Cientificos (CECs), \\ Av. Arturo Prat 514, Valdivia, Chile \\ E-mail: dmitry@iip.ufrn.br, fnovaes@iip.ufrn.br, aperez@cecs.cl, \\ troncoso@cecs.cl
}

ABSTRACT: Non-relativistic field theories with anisotropic scale invariance in $(1+1)-\mathrm{d}$ are typically characterized by a dispersion relation $E \sim k^{z}$ and dynamical exponent $z>1$. The asymptotic growth of the number of states of these theories can be described by an extension of Cardy formula that depends on $z$. We show that this result can be recovered by counting the partitions of an integer into $z$-th powers, as proposed by Hardy and Ramanujan a century ago. This gives a novel duality relationship between the characteristic energy of the dispersion relation with the cylinder radius and the ground state energy. For free bosons with Lifshitz scaling, this relationship is shown to be identically fulfilled by virtue of the reflection property of the Riemann $\zeta$-function. The quantum Benjamin-Ono $2\left(\mathrm{BO}_{2}\right)$ integrable system, relevant in the AGT correspondence, is also analyzed. As a holographic realization, we provide a special set of boundary conditions for which the reduced phase space of Einstein gravity with a couple of $U(1)$ fields on $\mathrm{AdS}_{3}$ is described by the $\mathrm{BO}_{2}$ equations. This suggests that the phase space can be quantized in terms of quantum $\mathrm{BO}_{2}$ states. Indeed, in the semiclassical limit, the ground state energy of $\mathrm{BO}_{2}$ coincides with the energy of global $\mathrm{AdS}_{3}$, and the Bekenstein-Hawking entropy for BTZ black holes is recovered from the anisotropic extension of Cardy formula.

Keywords: Integrable Field Theories, Space-Time Symmetries, Classical Theories of Gravity, Gauge-gravity correspondence

ARXIV EPRINT: 1808.04034 


\section{Contents}

1 Introduction 1

2 Microstate counting from number theory 4

3 Free boson with Lifshitz scaling $\quad 6$

4 Microstate counting and the quantum Benjamin-Ono 2 hierarchy $\quad 7$

$\begin{array}{lll}4.1 & \text { Classical formulation of the } \mathrm{BO}_{2} \text { hierarchy } & 7\end{array}$

$\begin{array}{ll}4.2 \text { Quantum } \mathrm{BO}_{2} \text { hierarchy } & 9\end{array}$

5 Geometrization of Benjamin-Ono 2 and black hole entropy in 3D

\section{Introduction}

Non-relativistic field theories in $(1+1)$-dimensions, possessing anisotropic Lifshitz scaling of the form

$$
t \rightarrow \lambda^{z} t, x \rightarrow \lambda x
$$

are typically characterized by modes with a dispersion relation $E \sim k^{z}$ and entropy $S \sim$ $E^{\frac{1}{z+1}}$, with dynamical exponent $z>1$. They have been extensively studied in the context of the holographic AdS/CMT correspondence, see e.g. [1-4]. A number of condensed matter systems are known to enjoy this type of scaling. For instance, the quantum Hall fluid has been proposed to possess a non-linear dispersion with $z=2[5,6]$, while the $(1+1)$-dimensional Bose gas in cold atom systems can be related to $z=3$ dispersion [7]. The non-relativistic effective field theory description of such systems is invariant under the Lifshitz group, which in $(1+1)$ - $\mathrm{d}$ is generated by translations in space and time and the anisotropic scale transformation (1.1). At finite temperature, chiral movers with Lifshitz scaling can be described in terms of the torus partition function

$$
Z[\tau ; z]=\sum_{E} \rho_{z}(E) e^{2 \pi \ell i \tau E},
$$

where $\tau=i \beta /(2 \pi \ell)$ is the modular parameter, defined in terms of the inverse temperature $\beta$ and the radius of the cylinder $\ell$, and $\rho_{z}(E)$ is the density of states at fixed energy $E$. As explained in $[8,9]$, assuming modular invariance of a well-defined partition function $Z[\tau ; z]$ in the anisotropic case implies that

$$
Z[\tau ; z]=Z\left[i^{1+\frac{1}{z}} \tau^{-\frac{1}{z}} ; z^{-1}\right] .
$$

Thus, eq. (1.3) can be regarded as the defining property of the partition functions of the theories we are interested in. Note that in the case of isotropic scaling $(z=1)$, eq. (1.3) reduces to the well-known modular invariance in $\mathrm{CFT}_{2}$ [10]. 
In the partition function $Z[\tau ; z]$, the modular parameter of the torus $\tau$ plays the standard role as a chemical potential, while the dynamical exponent $z$ turns out to be a parameter without variation which possesses a well-defined transformation property under a modular transformation. ${ }^{1}$

It is worth highlighting that in eq. (1.3), the existence of two different theories with different dynamical exponents $(z$ and $1 / z)$ that map into each other under a modular transformation is implicitly assumed. Thus, modular invariance of the partition function in the anisotropic case, as in eq. (1.3), relates the high and low energy spectrum of the corresponding Hamiltonians.

If one further assumes that the spectrum of the theory described by a dynamical exponent $z$ possesses a gap with a non-vanishing ground state energy given by $-E_{0}[z]$, then the asymptotic growth of the number of states at fixed energy $E \gg\left|E_{0}[z]\right|$ can be obtained from the inverse Laplace transform of (1.3) in the steepest descent approximation

$$
\rho_{z}(E) \approx \exp \left[2 \pi \ell(1+z)\left(\frac{\left|E_{0}\left[z^{-1}\right]\right|}{z}\right)^{\frac{z}{1+z}} E^{\frac{1}{1+z}}\right]
$$

and hence

$$
S=\log \rho_{z}(E)=2 \pi \ell(1+z)\left(\frac{\left|E_{0}\left[z^{-1}\right]\right|}{z}\right)^{\frac{z}{1+z}} E^{\frac{1}{1+z}},
$$

stands for the leading term of the microcanonical entropy. Note that for $z=1$, the entropy reduces to the well-known Cardy formula in $\mathrm{CFT}_{2}$ [18]. The logarithmic correction to (1.5) was discussed in [19] (see also [20]).

It is worth emphasizing that the high/low temperature duality of the partition function expressed by (1.3) can be argued to emerge from the combination of two purely geometric properties that a generic field theory with anisotropic scaling defined on a torus should possess. Indeed, on one hand, the lattice that corresponds to a generic torus is invariant under S-duality which swaps both periods preserving the orientation (see e.g. [21]). Besides, Lifshitz algebras in $2 \mathrm{~d}$ with dynamical exponents $z$ and $1 / z$ turn out to be isomorphic, since they are related by a change of basis in which the generators of space and Euclidean time translations are swapped [8]. Hence, the partition function of a theory possessing Lifshitz scaling that can be consistently defined on a torus should be, in particular, invariant under the combined action of S-duality and the isomorphism aforementioned. Consistency of both operations then provides evidence about the existence of a suitable dual theory described by a dynamical exponent $z^{-1}$, so that the partition function could be assumed to possess the duality property in eq. (1.3). In turn, it would be interesting to explore the possibility ${ }^{2}$ that the high/low temperature duality relationship applied to the theories described by

\footnotetext{
${ }^{1}$ Note that $z$ plays a similar role as that of the parameter $\lambda$ that characterizes the $T \bar{T}$ deformations of CFTs (see e.g. [11-15]). Indeed, $\lambda$ is not varied in the partition function and it also possesses a precise transformation property under modular transformations $[16,17]$. Modular invariance of the original $\mathrm{CFT}_{2}$ is recovered for $\lambda=0$, while in our case it does for $z=1$.

${ }^{2}$ We thank an anonymous referee for this suggestion.
} 
$z>1$ could actually be used to define the corresponding dual theories with dynamical exponent $z^{-1}$.

Hitherto, strong support to this high/low temperature duality can be gathered from a number of holographic examples, formulated in terms of different gravitational theories in three spacetime dimensions. In all cases, the black hole entropy, which is not necessarily given by a quarter of the event horizon area, is precisely recovered from (1.5) provided that $E$ and $E_{0}$ correspond to the (left/right) energies of the black hole and the ground state, respectively. The ground state configuration is given by a soliton, which turn out to be diffeomorphic to the black hole provided that the modular parameters of the corresponding tori at the boundary are related through the combination of S-duality and anisotropic scaling, precisely as in (1.3). Different cases of asymptotically Lifshitz black holes and their corresponding solitons were discussed in [8] (see also [22]), and in [23, 24]; while a different class of examples in which the anisotropic scaling is induced by special choices of boundary conditions were discussed in [9] and in [19, 25] (see also [26]).

The appearance of the absolute value of the ground state energy $\left|E_{0}\left[z^{-1}\right]\right|$ in (1.4) and (1.5), which precisely means taking the absolute value of the ground state energy $-E_{0}[z]$ and then evaluating it at $z^{-1}$, deserves some explanation. As discussed in [9], for certain special values of $z$, e.g. $z=4 n-1$, the (Lorentzian) ground state energy turns out to be positive. In these cases, in the Euclidean continuation, the corresponding holographic black hole configuration becomes diffeomorphic to the solitonic ground state, but with reversed orientation. This is due to the fact that the lapse function of an ADM foliation of the spacetime that describes the ground state reverses its sign. From the point of view of a thermal field theory in 2D with anisotropic Lifshitz scaling, that is defined on a torus, the lapse function can always be reabsorbed by the modular parameter (see e.g. [27, 28]). Thus, in these special cases, the high temperature configuration is also expected to be related to the ground state through S-duality. The direct implementation of this latter relationship in the $2 \mathrm{D}$ field theory is not entirely clear, but nonetheless, being inspired from the results in the bulk, the procedure can be interpreted in the following way: in the special cases, the S-modular transformation should incorporate some additional suitable operation that reverses the sign of the thermal period, which then also has the effect of flipping the sign of the ground state energy. Thus, in these cases, the suitable quantity that enters into (1.4) and (1.5) is $\left|E_{0}\left[z^{-1}\right]\right|$. This subtlety can be particularly well visualized in a concrete example we provide in section 3 when $z=2 m+1$. Indeed, for odd values of $m$, the ground state energy determined by $-E_{0}[z]$ in (3.4), turns out to be manifestly positive.

In the next section, we provide further evidence for the validity of this high/low temperature duality from a completely different approach, in which the microscopic counting of the states is performed applying some not so well-known results from number theory, developed a century ago by Hardy and Ramanujan. Consistency then provides a novel and nontrivial duality relation between the characteristic energy of the dispersion relation with the cylinder radius and the ground state energy.

In section 3, we consider free bosons with Lifshitz scaling in $2 \mathrm{~d}$, and we show that the duality relationship aforementioned turns out to be identically fulfilled, in a nontrivial way, by virtue of the reflection property of the Riemann $\zeta$-function. Thus, this example 
provides strong evidence supporting the fact that the high/low temperature duality can be explicitly realized directly in $2 \mathrm{~d}$ without the need of holographic setups.

Section 4 is devoted to the analysis of the quantum Benjamin-Ono $\left(\mathrm{BO}_{2}\right)$ integrable system, which possesses anisotropic Lifshitz scaling and also turns out to be relevant in the AGT correspondence. In particular, we show that in the semiclassical limit, for $z$ even, the spectrum becomes dominated by descendants, so that the characteristic energy can be precisely identified, and hence, the entropy can be obtained along the lines of number theory. In the case of $z$ odd, one is able to obtain the value of the ground state energy, and the entropy is consequently obtained by means of the anisotropic extension of Cardy formula.

Finally, relying on the results mentioned above, in section 5 we provide an explicit holographic realization of $\mathrm{BO}_{2}$, in which the semiclassical limit can be suitably taken. Concretely, we propose a new set of boundary conditions for which the reduced phase space of Einstein gravity with a couple of $\mathrm{U}(1)$ fields on $\mathrm{AdS}_{3}$ turns out to be described by the $\mathrm{BO}_{2}$ equations. Noteworthy, this example suggests that the phase space of the gravitational theory in the bulk can be quantized in terms of quantum $\mathrm{BO}_{2}$ states. Indeed, the ground state energy of quantum $\mathrm{BO}_{2}$, in the semiclassical limit, is shown to exactly coincide with the energy of global $\mathrm{AdS}_{3}$. This fact allows to precisely recovering the Bekenstein-Hawking entropy for BTZ black holes in terms of the anisotropic extension of Cardy formula in (1.5).

\section{Microstate counting from number theory}

In this section, we show that for $(1+1)$-dimensional weakly-coupled systems at high temperatures on a cylinder of radius $\ell$, the leading term of the asymptotic growth of the number of states $\rho_{z}(E)$ in (1.5) agrees with the asymptotic growth of the number of partitions of an integer $N$ into $z$-th powers $p_{z}(N)$. This is so provided that the ground state energy and the radius of the cylinder are precisely linked with the characteristic energy of the quasiparticles. Indeed, if the interactions are weak enough so that at high temperature the system behaves as a gas of free quasiparticles, as pointed out in the introduction, the dispersion relation has to be of the form

$$
E_{n}=\varepsilon_{z}\left(k_{n} \ell\right)^{z}=\varepsilon_{z} n^{z}
$$

where $k_{n}=n / \ell$ is the momentum, $n$ is a non-negative integer and $\varepsilon_{z}$ stands for the characteristic energy of the modes. The total energy is then given by

$$
E=\sum_{i} E_{n_{i}}=\varepsilon_{z} \sum_{i} n_{i}^{z}=\varepsilon_{z} N
$$

Therefore, assuming the ordering $n_{1} \geq n_{2} \geq \ldots \geq 0$ to count only indistinguishable configurations, the number of states with fixed energy $E$ corresponds to the combinatorial problem of finding the number of power partitions $p_{z}(N)$ for fixed $N=\sum_{i} n_{i}^{z}=E / \varepsilon_{z}$. Quite remarkably, this problem was solved in 1918 by Hardy and Ramanujan [29]. Indeed, in one of the last formulas of their paper, one finds that for large $N$, the leading term of 
the asymptotic growth of power partitions is given by

$$
p_{z}(N) \approx \exp \left[(1+z)\left(\frac{\Gamma\left(1+\frac{1}{z}\right) \zeta\left(1+\frac{1}{z}\right)}{z}\right)^{\frac{z}{1+z}} N^{\frac{1}{1+z}}\right] .
$$

Surprisingly, for a generic $z>1$ the result was actually a conjecture, proven later by Wright in 1934 using generalized Bessel functions [30]. A simplified proof has been recently given in [31] for $z=2$, and extended to $z \geq 2$ in [32], both using the Hardy-Littlewood circle method. Hence, at high temperature, the leading term of the entropy can be read from (2.2), and it is given by

$$
S=\log p_{z}(N)=(1+z)\left(\frac{\Gamma\left(1+\frac{1}{z}\right) \zeta\left(1+\frac{1}{z}\right)}{z}\right)^{\frac{z}{1+z}}\left(\frac{E}{\varepsilon_{z}}\right)^{\frac{1}{1+z}} .
$$

One then concludes that at high temperature, the asymptotic growth of the number of states obtained from anisotropic modular invariance, given by $\rho_{z}(E)$ in (1.4), agrees with the one from number theory given by $p_{z}(N)$ in $(2.2)$, provided that the characteristic energy of the dispersion relation is related to the radius of the cylinder and the non-vanishing ground state energy according to

$$
\left(\varepsilon_{\frac{1}{z}}\right)^{z}=\frac{\Gamma(1+z) \zeta(1+z)}{(2 \pi \ell)^{1+z}} \frac{1}{\left|E_{0}[z]\right|} .
$$

Note that, for $z=1$, the characteristic energy is related to the effective central charge as $c_{\text {eff }}=\left(\varepsilon_{1} \ell\right)^{-1}$, so that according to (2.4), the energy of the ground state acquires the expected form for chiral movers

$$
\left|E_{0}[1]\right|=\frac{c_{\mathrm{eff}}}{24 \ell}=\frac{1}{24 \ell^{2} \varepsilon_{1}} .
$$

It is worth emphasizing that the ability to express the leading term of the entropy in terms of the characteristic energy as in (2.3) possesses an advantage, since it opens up the possibilities to perform the microscopic counting even if the ground state energy vanishes.

Besides, if the ground state energy does not vanish, expressing the entropy as in (1.5) certainly helps, since its value can be directly obtained in cases where the microscopic counting cannot be explicitly performed.

Therefore, by virtue of (2.4), the high/low temperature duality for systems with anisotropic scaling not only acquires additional support, but it becomes enhanced from solid results in number theory.

An additional interesting remark is in order. Note that the asymptotic growth of the number of states in (1.4), which was obtained from modular invariance in the anisotropic case, actually holds for arbitrary real values of $z>0 .^{3}$ Therefore, by virtue of the equivalence of both ways of computing the entropy, expressed in (1.5) and (2.3), respectively,

\footnotetext{
${ }^{3}$ As pointed out in $[9,19,25]$, in the limit $z \rightarrow 0$ there is also a very intriguing link with the results in [26] about "soft hair" in the sense of Hawking, Perry and Strominger [33, 34].
} 
one is naturally led to conjecture that the expression for the asymptotic growth of the power partitions of Hardy and Ramanujan can actually be extended to hold for positive real values of $z$. The partition problem in this case can be then naturally defined as $E / \varepsilon_{z}=N=\sum_{i}\left\lfloor n_{i}^{z}\right\rfloor$, where $\lfloor x\rfloor$ stands for the floor of $x$. Indeed, very recent results in number theory give support to this conjecture, since it has already been proved for $z=\frac{1}{2}$ in [35] and for $0<z<1$ in [36]. Remarkably, Li and Chen in [36] have also arrived to the same conjecture, but following a completely different line of reasoning.

\section{Free boson with Lifshitz scaling}

In order to test the results of the previous section, it is instructive to consider the simple case of a free boson with Lifshitz scaling [37, 38] (see also e.g. [2, 4]), described by

$$
I=\frac{1}{2} \int d t d x\left[\left(\partial_{t} \varphi\right)^{2}-\sigma^{2(z-1)}\left(\partial_{x}^{z} \varphi\right)^{2}\right]
$$

with $0 \leq x<2 \pi \ell$. Here $\sigma$ is an arbitrary parameter with unit of length, and the units have been chosen such that, for $z=1$, the speed of light is unity. The dispersion relation of the modes then reads

$$
E_{n}= \pm \varepsilon_{z}|n|^{z}, \quad \varepsilon_{z}=\frac{\sigma^{z-1}}{\ell^{z}}
$$

so that the characteristic energy of left and right movers matches eq. (2.1). The Hamiltonian of chiral movers can then be written as

$$
H_{z}=2 \frac{\sigma^{z-1}}{\ell^{z}} \sum_{n>0} n^{z-1} a_{-n} a_{n}-E_{0}[z],
$$

where $\left[a_{n}, a_{m}\right]=\frac{n}{2} \delta_{n,-m}$, and by virtue of $\zeta$-function regularization, the ground state energy is determined by

$$
E_{0}[z]=-\frac{1}{2} \frac{\sigma^{z-1}}{\ell^{z}} \zeta(-z)
$$

Note that when $z$ takes odd values, the ground state energy (3.4) becomes non-trivial, and remarkably, the duality relation between the characteristic energy $\varepsilon_{z}$, the energy of the ground state $E_{0}[z]$ and the radius of the cylinder $\ell$ in (2.4) becomes identically fulfilled by the reflection property of the Riemann $\zeta$-function

$$
\zeta(-z)=-\frac{1}{2^{z} \pi^{1+z}} \sin \left(\frac{\pi z}{2}\right) \Gamma(1+z) \zeta(1+z) .
$$

Hence, for odd values of $z$, the leading term of the entropy can be either obtained from the number theory counting as in (2.3) with $\varepsilon_{z}=\frac{\sigma^{z-1}}{\ell^{z}}$, or equivalently by virtue of the expression obtained from the high/low temperature duality in (1.5) with $E_{0}[z]$ given by (3.4).

Therefore, the free boson with Lifshitz scaling for odd values of $z$ certainly appears to be an explicit example for which the high/low temperature duality can be manifestly realized in $2 \mathrm{~d}$.

For the case of even values of $z$, an interesting remark is in order. Indeed, in this case, one of the hypotheses assumed in order to derive the asymptotic growth of the number 
of states from modular invariance in the anisotropic case in (1.5) is not fulfilled, since the ground state energy in (3.4) manifestly vanishes for $z=2 n$. Nonetheless, in this case one is able to precisely identify the characteristic energy of the dispersion relation $\varepsilon_{z}$ to be given by (3.2), and hence, the entropy can still be directly obtained from the number theory counting given by $(2.3)$.

For a generic value of $z$, it is also worth pointing out that according to number theory, the sequence of power partitions possesses the following generating function (see e.g. $[29,32])$

$$
\sum_{N=0}^{\infty} p_{z}(N) q^{N}=\prod_{n=1}^{\infty} \frac{1}{1-q^{n^{z}}},
$$

and hence, the partition function for free bosons with Lifshitz scaling acquires the form

$$
Z[\tau ; z]=\mathcal{N}(\tau ; z)\left|q^{-E_{0}[z]} \prod_{n=1}^{\infty} \frac{1}{1-q^{n^{z}}}\right|^{2}
$$

with $q=e^{2 \pi i \tau}$. Here $\mathcal{N}(\tau ; z)$ stands for a non-exponential factor coming from the contribution of zero modes, so that it does not modify the leading high temperature asymptotics of $Z[\tau ; z]$. Subleading corrections and further details about its precise form in connection with modular invariance in the anisotropic case will be addressed in [39].

Note that for $z=1$, the action (3.1) reduces to the one of a free boson in $\mathrm{CFT}_{2}$, while the energy of the ground state energy is recovered from (3.4) to be given by $-E_{0}[1]=-\frac{1}{24 \ell}$, in agreement with the known result for chiral movers. Thus, the duality relation in (2.4) reduces to $(2.5)$, which is consistent with the fact that $c_{\mathrm{eff}}=1$. The suitable factor of the partition function in this case is given by $\mathcal{N}(\tau ; 1)=\operatorname{Im}(\tau)^{-1 / 2}$ (see e.g. [10]).

As an ending remark of this section, we would like to emphasize that the analysis of the free boson with Lifshitz scaling performed here, including its statistical mechanics, as far as the authors' knowledge, is new in the literature.

\section{Microstate counting and the quantum Benjamin-Ono 2 hierarchy}

In the previous section, we discussed a simple free bosonic model with Lifshitz scaling $z$ and how its spectrum is connected to the partitions of integers into $z$-th powers. Here we describe a quantum integrable system, with an infinite set of conserved quantities, presenting Lifshitz scaling in the semiclassical limit, the quantum Benjamin-Ono 2 model. This will give an interesting link between the semiclassical limit of quantum systems, microstate counting of models with Lifshitz scaling and gravitation on $\mathrm{AdS}_{3}$.

\subsection{Classical formulation of the $\mathrm{BO}_{2}$ hierarchy}

The Benjamin-Ono equation describes deep inner waves in a stratified fluid, being then a counterpart of the KdV equation for propagation in a shallow depth channel [40]. Both equations possess solitonic solutions and an infinite set of commuting conserved quantities, so that they belong to a hierarchy of integrable systems. 
The Benjamin-Ono 2 hierarchy is a generalization of these integrable systems [41-43], describing non-linear perturbations and solitonic waves on the edge of the quantum Hall fluid $[5,6,44,45]$, as well as in further applications of one-dimensional condensed matter systems $[5,45,46]$. It is defined in terms of two dynamical fields, $\mathcal{L}(t, \phi)$ and $\mathcal{J}(t, \phi)$, which we assume to be $2 \pi$-periodic in the $\phi$ coordinate.

The $\mathrm{BO}_{2}$ hierarchy also possesses solitonic solutions and an infinite set of commuting integrals of motion $H_{j}=\frac{c}{12 \pi \ell} \int \mathcal{H}_{j}(\phi) d \phi$, with $j \in \mathbb{Z}_{>0}$, so that the field equations of the $z$-th representative can be written in Hamiltonian form as

$$
\dot{\mathcal{L}}=\left\{\mathcal{L}, H_{z}\right\}, \quad \dot{\mathcal{J}}=\left\{\mathcal{J}, H_{z}\right\}
$$

where the Poisson brackets are given by ${ }^{4}$

$$
\begin{aligned}
& \{\mathcal{L}(\phi), \mathcal{L}(\bar{\phi})\}=-\frac{48 \pi}{c} \mathcal{D}_{\phi} \delta(\phi-\bar{\phi}), \quad\{\mathcal{L}(\phi), \mathcal{J}(\bar{\phi})\}=0, \\
& \{\mathcal{J}(\phi), \mathcal{J}(\bar{\phi})\}=\frac{6 \pi}{c} \partial_{\phi} \delta(\phi-\bar{\phi})
\end{aligned}
$$

and $\mathcal{D}_{\phi}=\partial_{\phi} \mathcal{L}+2 \mathcal{L} \partial_{\phi}-2 \partial_{\phi}^{3}$. The first three densities $\mathcal{H}_{j}$ read

$$
\begin{aligned}
& \mathcal{H}_{1}=\frac{1}{4} \mathcal{L}-\mathcal{J}^{2}, \quad \mathcal{H}_{2}=\frac{2}{3}\left[\frac{1}{4} \mathcal{L} \mathcal{J}-\frac{1}{3} \mathcal{J}^{3}-\mathcal{J} \mathrm{H} \partial_{\phi} \mathcal{J}\right] \\
& \mathcal{H}_{3}=\frac{1}{8} \mathcal{L}^{2}-3 \mathcal{L} \mathcal{J}^{2}-3 \mathcal{L} \mathrm{H} \partial_{\phi} \mathcal{J}+10\left(\partial_{\phi} \mathcal{J}\right)^{2}+12 \mathcal{J}^{2} \mathrm{H} \partial_{\phi} \mathcal{J}+2 \mathcal{J}^{4},
\end{aligned}
$$

where $\mathrm{H}$ is the Hilbert transform defined by the principal value integral

$$
\mathrm{H} F(\phi)=\frac{1}{2 \pi} \mathcal{P} \int_{0}^{2 \pi} F(\bar{\phi}) \cot \frac{1}{2}(\bar{\phi}-\phi) d \bar{\phi}
$$

The remaining conserved quantities of the hierarchy can be obtained recursively by imposing commutativity, $\left\{H_{k}, H_{l}\right\}=0$. More powerful methods to obtain the integrals of motion can be found in [41-43, 47, 48]. An action principle for the $\mathrm{BO}_{2}$ hierarchy can be formally written in terms of a non-local symplectic form given by the inverse of the Poisson brackets in (4.2), see e.g. [49-51].

For our purposes, it is worth stressing that the $\mathrm{BO}_{2}$ equations are invariant under anisotropic scaling of Lifshitz type with dynamical exponent $z$ in (1.1), provided that the fields scale as $\mathcal{L} \rightarrow \lambda^{-2} \mathcal{L}, \mathcal{J} \rightarrow \lambda^{-1} \mathcal{J}$. Indeed, the conserved charges scale according to $H_{j} \rightarrow \lambda^{-j} H_{j}$, and thus, the Hamiltonian of the $z$-th representative of the hierarchy, $H_{z}$, becomes labeled in terms of its scaling dimension $z$, which matches the dynamical exponent used before.

In spite of the non-locality introduced through the Hilbert transform, it is remarkable that $\mathrm{BO}_{2}$ can be quantized.

\footnotetext{
${ }^{4}$ In our conventions, the fields are expanded in modes according to (4.5), so that $c$ stands for the Virasoro central charge.
} 


\subsection{Quantum $\mathrm{BO}_{2}$ hierarchy}

The quantum $\mathrm{BO}_{2}$ hierarchy surprisingly emerges in the context of the AGT correspondence, which describes a relationship between $4 \mathrm{~d} \mathcal{N}=2$ supersymmetric gauge theories and $2 \mathrm{~d}$ conformal field theories [52]. The partition function of certain type of supersymmetric models, called class- $\mathcal{S}$ models, is given by the Nekrasov partition function $Z_{\text {inst }}$ [53]. The AGT correspondence states that $Z_{\text {inst }} \propto \mathcal{F}_{c}$, where $\mathcal{F}_{c}$ is a Liouville CFT conformal block. For the detailed map between the two sides, see [52]. Here, we just sketch the minimal information about the correspondence that is useful for our requirements.

The proof of the AGT expansion relies on the introduction of a new basis of descendant $\mathrm{CFT}_{2}$ states, which we call the AFLT basis [54]. It starts by considering the tensor product algebra $\mathcal{A}=\operatorname{Vir} \otimes \mathcal{H}$, spanned by the modes $L_{n}$ of the Virasoro algebra (Vir) and the modes $a_{n}$ of the Heisenberg algebra $(\mathcal{H})$, so that $\left[a_{n}, L_{m}\right]=0$. In CFT, the generators of $\mathcal{A}$ are given in terms of the energy-momentum tensor $T$ and the $\mathrm{U}(1)$ current $J$. Here we use an alternative normalization, with respect to CFT, for the mode expansion of these currents

$$
\begin{aligned}
& \hat{\mathcal{L}}(\phi)=\frac{24}{c} \sum_{n=-\infty}^{\infty} L_{n} e^{-i n \phi}-1, \\
& \hat{\mathcal{J}}(\phi)=i \sqrt{\frac{24}{c}} \sum_{n=-\infty, n \neq 0}^{\infty} a_{n} e^{-i n \phi},
\end{aligned}
$$

to match the conventions set in the classical formulation in section 4.1. As in [48, 54], we also discard the zero mode of the $u(1)$ current. Notice that, for $\hat{\mathcal{L}}$ and $\hat{\mathcal{J}}$ to be Hermitian, we set $L_{n}^{\dagger}=L_{-n}$ and $a_{n}^{\dagger}=-a_{-n}$.

To proceed, we introduce the standard Liouville notation for the central charge and conformal dimensions [55]

$$
c=1+6 Q^{2}, \quad Q=b+\frac{1}{b}, \quad \Delta(P)=\frac{Q^{2}}{4}-P^{2},
$$

where $b$ is the Liouville parameter and the momentum $P$ labels the primary states. The orthogonal AFLT basis reads

$$
|P\rangle_{\vec{\lambda}}=\sum_{|\vec{\mu}|=|\vec{\lambda}|} C_{\vec{\lambda}}^{\mu_{1}, \mu_{2}}(P) a_{-\mu_{1}} L_{-\mu_{2}}|\Delta(P)\rangle
$$

where the first few coefficients $C_{\vec{\lambda}}^{\mu_{1}, \mu_{2}}(P)$ are given in [54]. Here $\vec{\lambda}=\left(\lambda_{1}, \lambda_{2}\right)$ corresponds to two integer partitions $\left.\lambda_{k}=\left\{\left(\lambda_{k}\right)_{1},\left(\lambda_{k}\right)_{2}, \ldots,\left(\lambda_{k}\right)_{n}\right)\right\}$, with $k=1,2$, and $\lambda_{1} \geq \lambda_{2} \geq$ $\cdots \geq \lambda_{n}$. One of the main conclusions of [54] is that the insertion of the completeness relation of the basis (4.8) in a CFT correlator gives the AGT conformal block expansion.

The AFLT basis $|P\rangle_{\vec{\lambda}}$ also diagonalizes an infinite set of mutually commuting operators $\mathbf{H}_{j}, j \in \mathbb{Z}_{>0}$, given by the quantum $\mathrm{BO}_{2}$ integrals of motion and their eigenvalues can be explicitly obtained [48, 54]. The quantum integrals of motion $\mathbf{H}_{j}$ lie in the universal 
enveloping algebra of $\mathcal{A}$. The first two of them read

$$
\begin{aligned}
& \mathbf{H}_{1}=\frac{1}{\ell}\left(L_{0}+2 \sum_{k=1}^{\infty} a_{-k} a_{k}-\frac{c+1}{24}\right), \\
& \mathbf{H}_{2}=\frac{4 i}{3 \ell} \sqrt{\frac{6}{c}}\left(\sum_{k=-\infty, k \neq 0}^{\infty} a_{-k} L_{k}+2 i Q \sum_{k=1}^{\infty} k a_{-k} a_{k}+\frac{1}{3} \sum_{i+j+k=0} a_{i} a_{j} a_{k}\right),
\end{aligned}
$$

while for odd values of $z=2 n-1$ one obtains

$$
\mathbf{H}_{2 n-1}=\frac{1}{n \ell}\left(\frac{24}{c}\right)^{n-1}\left(L_{0}^{n}+\cdots\right)
$$

where the ellipsis stands for non-zero modes. Note that we have defined the operators $\mathbf{H}_{j}$ to be Hermitian, so that the spectrum is real, and the classical integrals of motion $H_{j}$ obtained from the densities (4.3), can be recovered from (4.9) in the semiclassical limit $b \rightarrow 0$.

The $\mathrm{BO}_{2}$ eigenstates $|P\rangle_{\vec{\lambda}}$ obey $\mathbf{H}_{z}|P\rangle_{\vec{\lambda}}=E_{\vec{\lambda}}^{(z)}(P)|P\rangle_{\vec{\lambda}}$, with eigenvalues given by $E_{\lambda_{1}, \lambda_{2}}^{(z)}(P)=E_{\lambda_{1}}^{(z)}(P)+E_{\lambda_{2}}^{(z)}(-P)$. For a generic Hamiltonian $\mathbf{H}_{z}$, it was conjectured in [54] and [48] that the spectrum can be written as a sum of two eigenvalues of the CalogeroSutherland model plus some extra terms depending only on $\Delta$ and $c$. The CalogeroSutherland eigenvalues are given by $h_{\lambda, \mu}^{(z)}=h_{\lambda}^{(z)}(P)+h_{\mu}^{(z)}(-P)$, where

$$
h_{\lambda}^{(z)}(P)=\frac{2^{z}}{(1+z) \ell}\left(\frac{6}{b^{2} c}\right)^{\frac{z-1}{2}} \sum_{j=1}^{N_{r}}\left[\left(b P-\frac{b^{2}}{2}+\lambda_{j}+j b^{2}\right)^{z}-\left(b P-\frac{b^{2}}{2}+j b^{2}\right)^{z}\right] .
$$

We call $h_{\lambda, \mu}^{(z)}$ the descendant part of the spectrum. The descendant part can be obtained from the Bethe ansatz equations conjectured in [48] and proven by [56]. We denote the contribution to the energy due to primary states as $E^{(z)}(P)$, so that the ground state energy is given by $E_{0}^{(z)} \equiv E^{(z)}\left( \pm \frac{Q}{2}\right)$. The eigenvalues of the first four operators $\mathbf{H}_{z}$, in our normalization, are given by

$$
\begin{aligned}
& E_{\lambda, \mu}^{(1)}(P)=h_{\lambda, \mu}^{(1)}(P)+E^{(1)}(P), \quad E_{\lambda, \mu}^{(2)}(P)=\frac{1}{2} h_{\lambda, \mu}^{(2)}(P), \\
& E_{\lambda, \mu}^{(3)}(P)=-2 h_{\lambda, \mu}^{(3)}(P)-\frac{6}{c \ell}\left(1+b^{2}\right) N_{\lambda, \mu}+E^{(3)}(P), \\
& E_{\lambda, \mu}^{(4)}(P)=\frac{1}{2} h_{\lambda, \mu}^{(4)}(P)+\frac{18}{5} \frac{\left(1+b^{2}\right)}{c} h_{\lambda, \mu}^{(2)}(P),
\end{aligned}
$$

where $N_{\lambda, \mu}=|\lambda|+|\mu|$, and

$$
\begin{aligned}
& E^{(1)}(P)=\frac{1}{\ell}\left[\Delta(P)-\frac{c+1}{24}\right], \\
& E^{(3)}(P)=\frac{12}{c \ell}\left[\Delta(P)^{2}-\frac{c+5}{12} \Delta(P)+\frac{5 c^{2}+52 c+15}{2880}\right] .
\end{aligned}
$$


Note that for a generic heavy state, where $\Delta(P) \approx(c / 24) \mathcal{L}(P)$ in the semiclassical limit, for $z$ odd we have

$$
E^{(z)}(P)=\frac{c}{12 \ell} \frac{1}{1+z}[\mathcal{L}(P)]^{\frac{1+z}{2}} .
$$

This corresponds to a classical state with energy $E^{(z)}(P)=\left\langle\Delta(P)\left|\hat{H}_{z}\right| \Delta(P)\right\rangle$.

Knowing the spectrum then allows us to obtain the leading term of the entropy in the semiclassical limit. In the case of even values of $z$, the leading entropy can be obtained along the lines of number theory, while for odd values of $z$ it can be done through anisotropic modular invariance.

Entropy for $z=2 n$ : In the semiclassical limit $b \rightarrow 0$, as it occurs for $z=2,4$, we assume that the energy levels have only contributions from the descendant part, so that $E_{\lambda, \mu}^{(z)}(P) \sim h_{\lambda, \mu}^{(z)}(P)$ to leading order in $c$. From (4.10), if $P \ll b^{-1}$, we have that

$$
E_{\lambda, \mu}^{(z)}=\varepsilon_{z} \sum_{k}\left(\lambda_{k}^{z}+\mu_{k}^{z}\right)
$$

This corresponds to energies close to the CFT gap. For states in which $P \sim \sqrt{c}$, the situation is more complicated, but, for large enough partitions, the energies are still dominated by (4.14). The explicit values of the characteristic energies for $z=2,4$ can be read from (4.11) to be given by $\varepsilon_{2}=\frac{2}{3 \ell}$ and $\varepsilon_{4}=\frac{8}{5 \ell}$.

According to (4.14), the asymptotic growth of the number of states then goes as in section 2 , but extended to a two-colored system. Indeed, the $N$-colored entropy for systems with Lifshitz scaling in $(1+1)$-d can be readily obtain from (2.3) by the replacement $\varepsilon_{z} \rightarrow \varepsilon_{z} / N^{z}$, [39]. Therefore, in this case the entropy is given by (2.3) with $\varepsilon_{z} \rightarrow 2^{-z} \varepsilon_{z}$.

Entropy for $z=2 n-1$ : in this case, the energies are no longer dominated by the descendant part, but instead by the primary part $E^{(z)}(P)$. In the semiclassical limit, assuming that $\Delta \ll c$, the leading contribution of $E^{(z)}(P)$ comes from normal ordering of the leading term of the Hamiltonian, $\mathcal{H}_{z} \sim \hat{\mathcal{L}}^{\frac{1+z}{2}}$, so that the ground state energy reads

$$
E_{0}^{(z)}=\frac{(-1)^{\frac{1+z}{2}}}{1+z} \frac{c}{12 \ell}
$$

Hence, in this case the entropy is determined by (1.5) with $E_{0}[z]=E_{0}^{(z)}$.

In the next section, we connect the present discussion of the semiclassical $\mathrm{BO}_{2}$ spectrum with gravitation on $\mathrm{AdS}_{3}$ and black holes.

\section{Geometrization of Benjamin-Ono 2 and black hole entropy in 3D}

Following the lines of [9], here we show that the $\mathrm{BO}_{2}$ hierarchy of integrable systems can be fully geometrized, in the sense that its dynamics can be equivalently understood in terms of the evolution of spacelike surfaces and $\mathrm{U}(1)$ fields with vanishing field strength embedded in locally $\mathrm{AdS}_{3}$ spacetimes. Let us then consider the Einstein-Hilbert action with negative cosmological constant in 3D, endowed with a couple of noninteracting $\mathrm{U}(1)$ fields

$$
I=\frac{1}{16 \pi G} \int d^{3} x\left[\sqrt{-g}\left(R+2 \ell^{-2}\right)-2 \ell \epsilon^{\mu \nu \lambda}\left(A_{\mu}^{+} \partial_{\nu} A_{\lambda}^{+}-A_{\mu}^{-} \partial_{\nu} A_{\lambda}^{-}\right)\right],
$$


which agrees with the bosonic sector of $\mathcal{N}=(2,2)$ supergravity [57]. Note that since the $\mathrm{U}(1)$ fields are described by Chern-Simons actions, the spacetime metric does not acquire a back reaction due to their presence. Therefore, the field equations imply that spacetime is of negative constant curvature, carrying two independent $U(1)$ fields of vanishing field strength.

As done in [9] (see also [58]), it can be shown that there exists a precise set of boundary conditions, being such that, in the reduced phase space, the field equations obtained from (5.1) exactly reduce to (left and right copies of) $\mathrm{BO}_{2}$. This can be seen as follows. According to $[57,59]$, up to boundary terms, the action (5.1) can be written as the difference of two Chern-Simons actions, both with level $k=\ell / 4 G$, for independent $\mathrm{SL}(2, R) \times \mathrm{U}(1)$ gauge fields, so that the dreibein and the (dualized) spin connection are related to the $\mathrm{SL}(2, R)$ gauge fields as $A^{ \pm} \mathrm{SL}(2, R)=\omega \pm e \ell^{-1}$. We then have to specify the asymptotic structure of the fields. For simplicity we restrict the analysis to the left copy, since the extension to the remaining one is straightforward. It is useful to make a gauge choice as in $[27,28,60]$, so that the $\mathrm{SL}(2, R) \times \mathrm{U}(1)$ connection reads

$$
\mathcal{A}=g^{-1}(d+a) g,
$$

with $g=e^{\log (r / \ell) L_{0}}$. This gauge choice certainly simplifies our task, since the remaining analysis can be performed in terms of the auxiliary gauge field

$$
a=a_{t} d t+a_{\phi} d \phi
$$

which exclusively depends on $t, \phi$.

Thus, the asymptotic form of (5.3) is proposed to be given by

$$
\begin{aligned}
& a_{\phi}=L_{1}-\frac{1}{4} \mathcal{L} L_{-1}+\mathcal{J} J_{0}, \\
& a_{t}=\mu L_{1}-\frac{1}{4} \mu \mathcal{L} L_{-1}-\mu^{\prime} L_{0}+\frac{1}{2} \mu^{\prime \prime} L_{-1}-\frac{1}{8} \xi J_{0},
\end{aligned}
$$

where $\mu, \xi$ stand for Lagrange multipliers associated to the dynamical fields $\mathcal{L}, \mathcal{J}$ respectively. The boundary conditions then become fully specified only once the Lagrange multipliers are kept fixed at the boundary, located at a fixed value of the radial coordinate. Our choice of boundary conditions then consists in precisely fixing $\mu$ and $\xi$ in terms of the dynamical fields and their derivatives along $\phi$ according to

$$
\mu=\frac{48 \pi}{c} \frac{\delta H_{z}}{\delta \mathcal{L}}, \quad \xi=\frac{48 \pi}{c} \frac{\delta H_{z}}{\delta \mathcal{J}}
$$

where $H_{z}$ stands for the $z$-th conserved charge of $\mathrm{BO}_{2}$, with $c$ given by the Brown-Henneaux central charge $c=3 \ell / 2 G[61]$.

Since we are dealing with a Chern-Simons theory, the field equations imply that the $\mathrm{SL}(2, R) \times \mathrm{U}(1)$ connection $\mathcal{A}$ is locally flat, and by virtue of the gauge choice in (5.2), the field strength of the auxiliary gauge field (5.3) also vanishes. Therefore, the components of $a$ in (5.4) reduce to an $\mathrm{SL}(2, R) \times \mathrm{U}(1)$-valued Lax pair formulation of the $\mathrm{BO}_{2}$ hierarchy, so that the field equations in (4.1) can be compactly written as

$$
f=d a+a^{2}=0 .
$$


Therefore, two independent copies of the $\mathrm{BO}_{2}$ equations are precisely recovered from the reduced phase space of the three-dimensional field equations of (5.1) endowed with our choice of boundary conditions.

Furthermore, according to [9], the symmetries of the $\mathrm{BO}_{2}$ equations, spanned by the conserved quantities $H_{j}$, now emerge from the set of diffeomorphisms that preserve the asymptotic form of the gauge field. Noteworthy, in the geometric framework, the symmetries of $\mathrm{BO}_{2}$ become Noetherian, and hence, the infinite set of commuting conserved charges $H_{j}$ is precisely obtained from the corresponding surface integrals in the canonical approach [62]. ${ }^{5}$ In particular, the total energy of a three-dimensional configuration that fulfills our boundary conditions, including gravitation and the U(1) fields, is then given by the sum of left and right Hamiltonians of $\mathrm{BO}_{2}$, i.e., $E=Q\left[\partial_{t}\right]=H_{z}^{+}+H_{z}^{-}$.

In sum, the whole structure of classical $\mathrm{BO}_{2}$, including its phase space, the infinite number of commuting charges and its field equations, emerges from the reduced phase space of gravitation on $\mathrm{AdS}_{3}$ coupled to two $U(1)$ fields with our boundary conditions. Hence, this construction provides a gravitational dual of two noninteracting left and right $\mathrm{BO}_{2}$ movers, describing locally $\mathrm{AdS}_{3}$ spacetimes with anisotropic scaling induced by the choice of boundary conditions. Consequently, any solution of the $\mathrm{BO}_{2}$ equations can be mapped into a locally $\mathrm{AdS}_{3}$ spacetime with suitable $U(1)$ fields of vanishing field strength. In particular, one of the most trivial $\mathrm{BO}_{2}$ configurations, given by $\mathcal{J}^{ \pm}=0$ and $\mathcal{L}^{ \pm}=\ell^{-2}\left(r_{+} \pm r_{-}\right)^{2}$ constants, corresponds to the geometry of a BTZ black hole in vacuum [64, 65]. Note that in the geometric picture this configuration is clearly non-trivial because the event horizon has Hawking temperature and entropy, and its mass and angular momentum become well defined in terms of left and right $\mathrm{BO}_{2}$ energies

$$
H_{z}^{ \pm}\left[\mathcal{L}_{ \pm}\right]=\frac{c}{12 \ell} \frac{1}{1+z} \mathcal{L}_{ \pm}^{\frac{1+z}{2}}
$$

provided that $z=2 n-1$. Note that (5.6) agrees with (4.13).

This geometric realization suggests that the reduced gravitational phase space could be quantized in terms of two copies of $\mathrm{BO}_{2}$, so that the states would be given by the AFLT ones in (4.8). Indeed, two points are worth to be emphasized:

(i) The ground state energy of quantum $\mathrm{BO}_{2}$ in the semiclassical limit, given by $E_{0}^{(z)}$ in (4.15), exactly coincides with the one of the geometric configuration of lowest energy, determined by global $\mathrm{AdS}_{3}$ spacetime. Indeed, left and right energies of $\mathrm{AdS}_{3}$ correspond to (5.6) with $\mathcal{L}_{ \pm}=-1$, and hence

$$
\underbrace{H_{z}^{ \pm}[-1]}_{\mathrm{AdS}_{3}}=\underbrace{E_{0}^{(z)}}_{\text {Quantum } \mathrm{BO}_{2}}=\frac{(-1)^{\frac{1+z}{2}}}{1+z} \frac{c}{12 \ell} .
$$

\footnotetext{
${ }^{5}$ In the special case of $z=1$ our boundary conditions reduce to the bosonic part of the ones in [63], and the asymptotic symmetry algebra corresponds to two copies of the direct sum of Virasoro with the Brown-Henneaux central extension and a $u(1)$ current.
} 
(ii) The leading term of the asymptotic growth of the number of states is then obtained from (1.5) for both copies, i.e.,

$$
S=2 \pi \ell(1+z)\left[\left(\frac{\left|E_{0}^{+}\left[z^{-1}\right]\right|}{z}\right)^{\frac{z}{1+z}} E_{+}^{\frac{1}{1+z}}+\left(\frac{\left|E_{0}^{-}\left[z^{-1}\right]\right|}{z}\right)^{\frac{z}{1+z}} E_{-}^{\frac{1}{1+z}}\right],
$$

where $E_{0}^{+}[z]=E_{0}^{-}[z]$ stand for left and right energies of the ground state, determined by (5.7). Hence, for left and right energies given by the ones of the black hole, i.e., $E_{ \pm}=H_{z}^{ \pm}\left[\mathcal{L}_{ \pm}\right]$in $(5.6)$, noteworthy, the entropy obtained from the anisotropic extension of Cardy formula (5.8) exactly reduces to the one of Bekenstein and Hawking, given by

$$
S=\frac{A}{4 G}
$$

\section{Acknowledgments}

The authors thank valuable discussions with Sebas Eliens, Hernán González, Rodrigo Pereira, Miguel Pino, Pablo Rodríguez, David Tempo, Jacopo Viti, Paul Wiegmann and Alexander B. Zamolodchikov. The work of DM was supported by the grant No. 16-1210344 of the Russian Science Foundation. FN thanks Máté Lencsés for pointing out the Hardy-Ramanujan paper. FN also thanks Jun'ichi Shiraishi and, specially, Yohei Tutiya for the initial discussions on this project and kind hospitality at the Komaba Mathematics section of the University of Tokyo, where part of this work was developed. FN thanks the organizers of the Latin-American Workshop on Gravity and Holography held in São Paulo in June, 2018 for the opportunity to present the main results of this work and for financial support. FN acknowledges the Brazilian Ministry of Education for the financial support. AP thanks Stefan Theisen for his kind hospitality at the MPI für Gravitationsphysik in Golm, and the German Academic Exchange Service (DAAD) for financial support through the "Re-invitation Programme for Former DAAD Scholarship Holders". AP and RT thank Fondecyt grants $\mathrm{N}^{\circ} 1161311,1171162,1181031$ and 1181496 for financial support. The Centro de Estudios Científicos (CECs) is funded by the Chilean Government through the Centers of Excellence Base Financing Program of Conicyt.

Open Access. This article is distributed under the terms of the Creative Commons Attribution License (CC-BY 4.0), which permits any use, distribution and reproduction in any medium, provided the original author(s) and source are credited.

\section{References}

[1] M. Taylor, Non-relativistic holography, arXiv:0812.0530 [INSPIRE].

[2] S.A. Hartnoll, Lectures on holographic methods for condensed matter physics, Class. Quant. Grav. 26 (2009) 224002 [arXiv:0903.3246] [INSPIRE].

[3] S.A. Hartnoll, Horizons, holography and condensed matter, in Black holes in higher dimensions, G.T. Horowitz ed., (2012), pg. 387 [arXiv:1106.4324] [INSPIRE]. 
[4] M. Taylor, Lifshitz holography, Class. Quant. Grav. 33 (2016) 033001 [arXiv:1512.03554] [INSPIRE].

[5] E. Bettelheim, A.G. Abanov and P. Wiegmann, Quantum shock waves: the case for non-linear effects in dynamics of electronic liquids, Phys. Rev. Lett. 97 (2006) 246401 [cond-mat/0606778] [INSPIRE].

[6] P. Wiegmann, Non-linear hydrodynamics and fractionally quantized solitons at fractional quantum Hall edge, Phys. Rev. Lett. 108 (2012) 206810 [arXiv:1112.0810] [InSPIRE].

[7] S. Sotiriadis, Equilibration in one-dimensional quantum hydrodynamic systems, J. Phys. A 50 (2017) 424004 [arXiv:1612.00373] [InSPIRE].

[8] H.A. Gonzalez, D. Tempo and R. Troncoso, Field theories with anisotropic scaling in 2D, solitons and the microscopic entropy of asymptotically Lifshitz black holes, JHEP 11 (2011) 066 [arXiv:1107.3647] [INSPIRE].

[9] A. Pérez, D. Tempo and R. Troncoso, Boundary conditions for general relativity on AdS 3 and the KdV hierarchy, JHEP 06 (2016) 103 [arXiv: 1605.04490] [INSPIRE].

[10] P. Di Francesco, P. Mathieu and D. Senechal, Conformal field theory, Graduate Texts in Contemporary Physics, Springer-Verlag, New York, NY, U.S.A. (1997) [INSPIRE].

[11] A.B. Zamolodchikov, Expectation value of composite field $T \bar{T}$ in two-dimensional quantum field theory, hep-th/0401146 [INSPIRE].

[12] F.A. Smirnov and A.B. Zamolodchikov, On space of integrable quantum field theories, Nucl. Phys. B 915 (2017) 363 [arXiv: 1608. 05499] [inSPIRE].

[13] A. Cavaglià, S. Negro, I.M. Szécsényi and R. Tateo, TT̄-deformed $2 D$ quantum field theories, JHEP 10 (2016) 112 [arXiv:1608.05534] [INSPIRE].

[14] L. McGough, M. Mezei and H. Verlinde, Moving the CFT into the bulk with $T \bar{T}$, JHEP 04 (2018) 010 [arXiv: 1611.03470] [INSPIRE].

[15] P. Kraus, J. Liu and D. Marolf, Cutoff $A d S_{3}$ versus the TT deformation, JHEP 07 (2018) 027 [arXiv:1801.02714] [INSPIRE].

[16] S. Datta and Y. Jiang, TT deformed partition functions, JHEP 08 (2018) 106 [arXiv: 1806.07426] [INSPIRE].

[17] O. Aharony, S. Datta, A. Giveon, Y. Jiang and D. Kutasov, Modular invariance and uniqueness of TT deformed CFT, JHEP 01 (2019) 086 [arXiv:1808.02492] [INSPIRE].

[18] J.L. Cardy, Operator content of two-dimensional conformally invariant theories, Nucl. Phys. B 270 (1986) 186 [InSPIRE].

[19] D. Grumiller, A. Perez, D. Tempo and R. Troncoso, Log corrections to entropy of three dimensional black holes with soft hair, JHEP 08 (2017) 107 [arXiv:1705.10605] [INSPIRE].

[20] E. Shaghoulian, A Cardy formula for holographic hyperscaling-violating theories, JHEP 11 (2015) 081 [arXiv: 1504.02094] [INSPIRE].

[21] T.M. Apostol, Modular functions and Dirichlet series in number theory, Springer, New York, NY, U.S.A. (1990).

[22] E. Ayon-Beato, A. Garbarz, G. Giribet and M. Hassaine, Lifshitz black hole in three dimensions, Phys. Rev. D 80 (2009) 104029 [arXiv:0909.1347] [INSPIRE]. 
[23] E. Ayón-Beato, M. Bravo-Gaete, F. Correa, M. Hassaïne, M.M. Juárez-Aubry and J. Oliva, First law and anisotropic Cardy formula for three-dimensional Lifshitz black holes, Phys. Rev. D 91 (2015) 064006 [Addendum ibid. D 96 (2017) 049903] [arXiv:1501.01244] [INSPIRE].

[24] M. Bravo-Gaete, S. Gomez and M. Hassaine, Cardy formula for charged black holes with anisotropic scaling, Phys. Rev. D 92 (2015) 124002 [arXiv:1510.04084] [INSPIRE].

[25] H. Afshar, D. Grumiller, W. Merbis, A. Perez, D. Tempo and R. Troncoso, Soft hairy horizons in three spacetime dimensions, Phys. Rev. D 95 (2017) 106005 [arXiv:1611.09783] [INSPIRE].

[26] H. Afshar et al., Soft Heisenberg hair on black holes in three dimensions, Phys. Rev. D 93 (2016) 101503 [arXiv:1603.04824] [INSPIRE].

[27] M. Henneaux, A. Perez, D. Tempo and R. Troncoso, Chemical potentials in three-dimensional higher spin anti-de Sitter gravity, JHEP 12 (2013) 048 [arXiv:1309.4362] [INSPIRE].

[28] C. Bunster, M. Henneaux, A. Perez, D. Tempo and R. Troncoso, Generalized black holes in three-dimensional spacetime, JHEP 05 (2014) 031 [arXiv: 1404.3305] [INSPIRE].

[29] G.H. Hardy and S. Ramanujan, Asymptotic formula in combinatory analysis, Proc. Lond. Math. Soc. s2-17 (1918) 75.

[30] E.M. Wright, Asymptotic partition formulae. III. Partitions into $k$-th powers, Acta Math. 63 (1934) 143.

[31] R.C. Vaughan, Squares: additive questions and partitions, Int. J. Number Theor. 11 (2015) 1367.

[32] A. Gafni, Power partitions, J. Number Theor. 163 (2016) 19 [arXiv:1506.06124].

[33] S.W. Hawking, M.J. Perry and A. Strominger, Soft hair on black holes, Phys. Rev. Lett. 116 (2016) 231301 [arXiv: 1601.00921] [INSPIRE].

[34] S.W. Hawking, M.J. Perry and A. Strominger, Superrotation charge and supertranslation hair on black holes, JHEP 05 (2017) 161 [arXiv:1611.09175] [INSPIRE].

[35] F. Luca and D. Ralaivaosaona, An explicit bound for the number of partitions into roots, J. Number Theor. 169 (2016) 250.

[36] Y.-L. Li and Y.-G. Chen, On the $r$-th root partition function, II, J. Number Theor. 188 (2018) 392.

[37] E. Lifshitz, On the theory of second-order phase transitions I, Zh. Eksp. Teor. Fiz. 11 (1941) 255.

[38] E. Lifshitz, On the theory of second-order phase transitions II, Zh. Eksp. Teor. Fiz. 11 (1941) 269.

[39] D. Melnikov, F. Novaes, A. Pérez and R. Troncoso, work in progress.

[40] Y. Matsuno, Bilinear transformation method, volume 174, Academic Press, New York, NY, U.S.A. (1984).

[41] D.R. Lebedev and A.O. Radul, Generalized internal long waves equations: construction, Hamiltonian structure and conservation laws, Commun. Math. Phys. 91 (1983) 543 [INSPIRE]. 
[42] A. Degasperis, D. Lebedev, M. Olshanetsky, S. Pakuliak, A. Perelomov and P. Santini, Nonlocal integrable partners to generalized $M K d V$ and two-dimensional Toda lattice equations in the formalism of a dressing method with quantized spectral parameter, Commun. Math. Phys. 141 (1991) 133 [INSPIRE].

[43] A. Degasperis et al., Generalized intermediate long-wave hierarchy in zero-curvature representation with noncommutative spectral parameter, J. Math. Phys. 33 (1992) 3783.

[44] A.G. Abanov and P.B. Wiegmann, Quantum hydrodynamics, quantum Benjamin-Ono equation and Calogero model, Phys. Rev. Lett. 95 (2005) 076402 [cond-mat/0504041] [INSPIRE].

[45] A.G. Abanov, E. Bettelheim and P. Wiegmann, Integrable hydrodynamics of Calogero-Sutherland model: bidirectional Benjamin-Ono equation, J. Phys. A 42 (2009) 135201 [arXiv:0810.5327] [inSPIRE].

[46] A. Imambekov, T.L. Schmidt and L.I. Glazman, One-dimensional quantum liquids: beyond the Luttinger liquid paradigm, Rev. Mod. Phys. 84 (2012) 1253 [arXiv:1110.1374].

[47] V.V. Bazhanov, S.L. Lukyanov and A.B. Zamolodchikov, Integrable structure of conformal field theory, quantum KdV theory and thermodynamic Bethe ansatz, Commun. Math. Phys. 177 (1996) 381 [hep-th/9412229] [INSPIRE].

[48] A.V. Litvinov, On spectrum of ILW hierarchy in conformal field theory, JHEP 11 (2013) 155 [arXiv: 1307.8094] [INSPIRE].

[49] I.M. Gel'fand and I.Y. Dorfman, Hamiltonian operators and algebraic structures related to them, Funct. Anal. Appl. 13 (1980) 248.

[50] A. Das, Integrable models, World Sci. Lect. Notes Phys. 30 (1989) 1 [INSPIRE].

[51] P.J. Olver, Applications of Lie groups to differential equations, volume 107, Springer, U.S.A. (1986).

[52] L.F. Alday, D. Gaiotto and Y. Tachikawa, Liouville correlation functions from four-dimensional gauge theories, Lett. Math. Phys. 91 (2010) 167 [arXiv:0906.3219] [INSPIRE].

[53] N.A. Nekrasov, Seiberg-Witten prepotential from instanton counting, Adv. Theor. Math. Phys. 7 (2003) 831 [hep-th/0206161] [inSPIRE].

[54] V.A. Alba, V.A. Fateev, A.V. Litvinov and G.M. Tarnopolskiy, On combinatorial expansion of the conformal blocks arising from AGT conjecture, Lett. Math. Phys. 98 (2011) 33 [arXiv: 1012.1312] [INSPIRE].

[55] S. Ribault, Conformal field theory on the plane, arXiv:1406.4290 [INSPIRE].

[56] B. Feigin, M. Jimbo and E. Mukhin, Integrals of motion from quantum toroidal algebras, J. Phys. A 50 (2017) 464001 [arXiv:1705.07984] [inSPIRE].

[57] A. Achucarro and P.K. Townsend, Extended supergravities in $d=(2+1)$ as Chern-Simons theories, Phys. Lett. B 229 (1989) 383 [INSPIRE].

[58] O. Fuentealba et al., Integrable systems with $B M S_{3}$ Poisson structure and the dynamics of locally flat spacetimes, JHEP 01 (2018) 148 [arXiv:1711.02646] [INSPIRE].

[59] E. Witten, $(2+1)$-dimensional gravity as an exactly soluble system, Nucl. Phys. B 311 (1988) 46 [INSPIRE]. 
[60] O. Coussaert, M. Henneaux and P. van Driel, The asymptotic dynamics of three-dimensional Einstein gravity with a negative cosmological constant, Class. Quant. Grav. 12 (1995) 2961 [gr-qc/9506019] [INSPIRE].

[61] J.D. Brown and M. Henneaux, Central charges in the canonical realization of asymptotic symmetries: an example from three-dimensional gravity, Commun. Math. Phys. 104 (1986) 207 [INSPIRE].

[62] T. Regge and C. Teitelboim, Role of surface integrals in the Hamiltonian formulation of general relativity, Annals Phys. 88 (1974) 286 [INSPIRE].

[63] M. Henneaux, L. Maoz and A. Schwimmer, Asymptotic dynamics and asymptotic symmetries of three-dimensional extended AdS supergravity, Annals Phys. 282 (2000) 31 [hep-th/9910013] [INSPIRE].

[64] M. Bañados, C. Teitelboim and J. Zanelli, The black hole in three-dimensional space-time, Phys. Rev. Lett. 69 (1992) 1849 [hep-th/9204099] [INSPIRE].

[65] M. Bañados, M. Henneaux, C. Teitelboim and J. Zanelli, Geometry of the $(2+1)$ black hole, Phys. Rev. D 48 (1993) 1506 [Erratum ibid. D 88 (2013) 069902] [gr-qc/9302012] [INSPIRE]. 